www.jmscr.igmpublication.org

Impact Factor 5.84

Index Copernicus Value: 83.27

ISSN (e)-2347-176x ISSN (p) 2455-0450

crossref DOI: _https://dx.doi.org/10.18535/jmscr/v5i7.12

Journal Of Medical Science And Clinical Research

IGM Publication

An Official Publication of IGM Publication

\title{
Guillain Barre Syndrome- A Rare New Variant with Mixed Pathology And Unilateral Facial Nerve Palsy
}

\author{
Authors \\ Dr Nutan Kumar.D.M ${ }^{1}$, Dr Tirthankar Mukherjee ${ }^{2}$, Dr Ramachandra Prabhu H.D , $^{3}$ \\ Dr Nagesh G.N ${ }^{4}$ \\ ${ }^{1}$ Junior Resident, Department of Medicine, Kempegowda Institute of Medical Sciences, K.R Road, V.V \\ Puram, Bangalore- 560004, Contact No: +919844596676 \\ Email: nutankdm.3008@gmail.com \\ ${ }^{2}$ Assistant Professor, Department of Medicine, Kempegowda Institute of Medical Sciences, K.R Road, V.V \\ Puram, Bangalore- 560004, Contact No:+919980188793 \\ Email: doctor.tirthankar@gmail.com \\ ${ }^{3}$ Associate Professor, Department of Medicine, Kempegowda Institute of Medical Sciences, K.R Road, V.V \\ Puram, Bangalore- 560004, Contact No:+919845296673 \\ ${ }^{4}$ Professor, Department of Medicine, Kempegowda Institute of Medical Sciences, K.R Road, V.V Puram, \\ Bangalore- 560004, Contact No:+919535410888
}

\begin{abstract}
Guillain-Barre syndrome (GBS) is an autoimmune disorder manifested as acute, rapidly progressive ascending paralysis with usually bilateral cranial nerve involvement. Facial nerve is most commonly involved usually bilateral but rarely unilateral. Here we are reporting such a rare case of unilateral facial nerve palsy in GBS. The electrophysiological tests including blink study are important in such cases. Our patient had mixed pathological features of demyelinating polyneuropathy in motor nerves of upper / lower limbs and motor axonopathy in both facial nerve (left > right) which is a rare combination seen in GBS patients. This can be a new variant seen in GBS patients which is first of its kind.

Keywords: Guillain-Barre Syndrome, Axonopathy, Demyelinating polyneuropathy, Unilateral Facial Nerve Palsy, Blink Reflexes.
\end{abstract}

\section{INTRODUCTION}

Guillain-Barre syndrome (GBS) is an autoimmune demyelinating disorder which is characterised by acute onset progressive poly-radiculo-neuropathy. It manifests as acute, rapidly progressive symmetrical ascending paralysis of limbs and areflexia $^{1,2,3,4}$. It has many variants including acute inflammatory demyelinating polyneuropathy, acute motor axonopathy, acute sensory motor axonopathy, miller fischer, pure facial diplegia, paraparesis with facial diparesis. Facial nerve palsy is one of the characteristic feature of $\mathrm{GBS}^{5,6}$. Facial paresis in GBS is mostly bilateral but rarely it can manifest as unilateral clinically ${ }^{6}$. We report a rare case of an elderly woman who presented with unilateral facial paresis and paraparesis who was later diagnosed to have GBS with different pathology in facial nerve and lower limb nerves. 
The electro-physiological tests including blink study are of great importance to diagnose underlying facial nerve involvement in such cases.

\section{CASE HISTORY}

A 53 year old female presented with complaints of weakness of both lower limbs, difficulty in walking/getting up from squatting position since 5 days, tingling sensation in both lower limbs and hands since 5 days, deviation of angle of mouth to right and inability to close left eye since 1 day. Weakness was progressive, no bladder/bowel involvement. There was no history of any skin rash or tick bite in the past. No history of fever/sore throat recently. She was not a known diabetic/hypertensive. No history of any surgeries/trauma.

\section{General Examination}

Pulse-76/min, BP-120/80, RR-16cpm. Patient didn't have pallor /edema / icterus / cyanosis / clubbing / lymphadenopathy.

\section{Systemic Examination}

On examination, higher mental function was normal. Cranial nerve examination revealed left sided lower motor neuron type of facial palsy with difficulty to close left eye. Rest of the cranial nerve examination was normal. Motor system examination revealed hypotonia in bilateral lower limbs. Power was grade $3 / 5$ in lower limbs and $5 / 5$ in upper limbs. Deep tendon reflexes were $2+$ in upper limbs and absent in both lower limbs. Plantar reflex was bilaterally mute. Sensory system and cerebellar examination was normal. Signs of meningeal irritation were absent.Other system examination was normal.

\section{Picture -A}

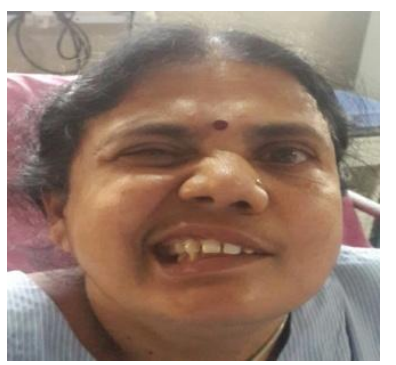

Patient photograph of the face revealing unilateral Left-sided facial palsy
Picture- B

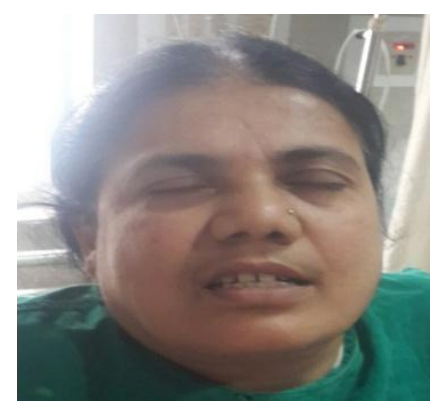

Patient photograph showing partial facial recovery

\section{INVESTIGATIONS}

- Complete haemogram was normal

- Blood sugar was normal

- HIV, HbsAg ELISA tests were non reactive

- Serum vitamin B12 level was 1476 which was normal

- CSF analysis showed Albuminocytological dissociation with protein82.3, cell count- 3(lymphocytes)

- MRI brain was normal.

- Motor Nerve conduction study revealed demyelinating polyradiculoneuropathy (Lower Limb>Upper Limb ) with increased distal latency and normal amplitude and absent $\mathrm{F}$ wave.

- Sensory nerve axon potential (SNAP) was normal in all limbs.

- Facial motor Nerve Conduction Study to facial orbicularis oculi and nasalis revealed bilateral facial axonopathy (left>right) with decreased amplitude and normal distal latency.

- Blink reflex revealed latency on both sides(left $>$ right)

Table: Facial Nerve Conduction Study

\begin{tabular}{|c|c|c|c|c|c|}
\hline MNCV & SitelSegment & Latency & Amplitude & Duration & Area \\
& & $\mathrm{ms}$ & $\mathrm{mV}$ & $\mathrm{ms}$ & $\mathrm{mV}$.ms \\
\hline Facial Occulai R & S1.0cculai & 5.7 & $\begin{array}{c}1.4 \\
-\end{array}$ & 11.1 & 6.8 \\
\hline Facial Nasalis R & Bel. Ear lobe-Nasalis & 4.5 & $\underline{1.0}$ & 15.8 & 5.4 \\
\hline Facial Occulai L & S1.0cculai & 5.8 & $\underline{1.3}$ & 10.3 & 5.0 \\
\hline Facial Nasalis L & Bel. Ear lobe-Nasalis & 3.8 & 1.4 & $\underline{11.8}$ & 4.9 \\
\hline
\end{tabular}




\section{DIAGNOSIS}

Patient was diagnosed with Guillain barre syndrome, clinically manifesting as lower motor neuron type of left facial nerve palsy and paraparesis. But electro-physiologically showing acute demyelinating polyneuropathy of all four limbs and axonopathy of both facial nerve.

\section{TREATMENT AND OUTCOME}

Patient was admitted in ICU and 5 cycles of plasmapheresis done following which facial palsy improved and patient was able to walk on $7^{\text {th }}$ day.

\section{DISCUSSION}

GBS is an acute, frequently severe and fulminant polyradiculoneuropathy that is autoimmune in nature. It occurs at the rate of 1 to 4 cases per 1 lakh annually. Males are at higher risk than females.GBS manifests as rapidly evolving areflexic ascending motor paralysis with or without sensory disturbances in form of tingling disesthesias. Legs are more affected than arms ${ }^{1,2}$. In comparison our patient had paraesthesia in all limbs with weakness of only lower limbs and not involving upper limbs. Bilateral facial nerve palsy is the most common pattern of cranial nerve involvement in GBS. However, unilateral facial palsy, although uncommon, can be seen in $\mathrm{GBS}^{7}$. A study conducted by Stephen L Hauser et al. showed that bilateral facial paralysis is present in $50 \%$ of patients with $\mathrm{GBS}^{1}$. On contrary unilateral facial nerve palsy was observed in studies conducted by Verma et al. and Kamihiro $\mathrm{N}$ et al. which showed right and left sided facial nerve palsy respectively ${ }^{6,7}$. In comparison our patient had left sided facial nerve palsy clinically.

A study by Sakakibara Y et al.showed acute left facial, hypoglossal, and phrenic nerve palsies, as well as dysphagia and weakness in the neck and arms with electrophysiologic studies of cranial nerves showing acute motor axonal neuropathy ${ }^{8}$. In our patient facial motor nerve conduction study to facial orbicularis oculi and nasalis revealed bilateral facial axonopathy (left $>$ right) with decreased amplitude and normal distal latency.
Thus showing axonopathy in facial nerve. This signifies the role of electrophysiological studies such as facial nerve conduction study and blink reflex in GBS to detect subclinical facial nerve involvement, if, clinically, patient has unilateral facial paralysis which may make it difficult to diagnose GBS $^{9}$.

Nerve conduction study of limbs showed delayed distal latency (lower limbs > upper limbs) with normal amplitude suggestive of demyelinating pathology but sensory nerve conduction study was normal with normal SNAP in all limbs inspite of presence of paraesthesias..

Treatment of GBS includes supportive care and disease modifying therapy with Intravenous Immune Globulin (IVIG) or Plasmapheresis. IVIG or Plasmapheresis therapy should be started within first two weeks of disease onset in patients who are not able to walk without support. There are no definite recommendations for patients with milder disease who can walk without support ${ }^{10}$.

In our patient Plasmapheresis was done for 5 cycles which improved lower limb and facial nerve weakness within one week.

Patient was not given immunoglobulins since there was improvement seen after 3 cycles of plasmapheresis.

\section{CONCLUSION}

Cranial nerves are affected in about $50 \%$ of GBS patients. Facial nerve palsy usually bilateral, is the most common cranial nerve palsy in GBS. Unilateral facial palsy, though rare, can be seen in GBS. Electrophysiological tests, including blink reflexes are of appreciable importance in such patient to identify subclinical involvement of facial nerve on the other side in such patients.

Our patient had mixed pathological features of demyelinating pathology in motor nerves of upper / lower limbs and axonopathy in both facial nerve (left > right) which is a rare combination seen in GBS patients. This can be a new variant seen in GBS patients which is first of its kind.

Competing interests: None

Patient consent: Obtained 


\section{REFERENCES}

1. Hauser SL, Asbury AK. Guillain-Barre syndrome and other immune-mediated neuropathies. harrisons principles of internal medicine. 2005;16(2):2513.

2. Asbury AK, Cornblath DR. Assessment of current diagnostic criteria for Guillain-Barré syndrome. Annals of neurology. 1990 Jan 1;27(S1).

3. Smith N, Grattan-Smith P, Andrews PI, Kainer G. Acquired facial palsy with hypertension secondary to Guillain-Barre syndrome. Journal of paediatrics and child health. 2010 Mar 1;46(3):125-7.

4. Ropper AH. The Guillain-Barré syndrome. New England Journal of Medicine. 1992 Apr 23;326(17):1130-6.

5. Winer JB. Guillain-Barré syndrome: Clinical variants and their pathogenesis. Journal of neuroimmunology. 2011 Feb 28;231(1):70-2.

6. Verma R, Chaudhari TS, Giri P. Unilateral facial palsy in Guillain-Barre syndrome (GBS): a rare occurrence. BMJ case reports. 2012 Oct 19;2012:bcr2012007077.

7. Kamihiro N, Higashigawa M, Yamamoto T, Yoshino A, Sakata K, Nashida Y, Maji $\mathrm{T}$, Fujiwara $\mathrm{T}$, Inoue $\mathrm{M}$. Acute motorsensory axonal Guillain-Barré syndrome with unilateral facial nerve paralysis after rotavirus gastroenteritis in a 2-year-old boy. Journal of Infection and Chemotherapy. 2012 Jan 1;18(1):119-23.

8. Sakakibara Y, Mori M, Kuwabara S, Katayama K, Hattori T, Koga M, Yuki N. Unilateral cranial and phrenic nerve involvement in axonal Guillain-Barré syndrome. Muscle \& nerve. 2002 Feb 1;25(2):297-9.

9. Crone C, Krarup C. Diagnosis of acute neuropathies. Journal of neurology. 2007 Sep 1;254(9):1151-69.

10. Kumar P, Charaniya R, Bahl A, Ghosh A, Dixit J. Facial Diplegia with Paresthesia: An Uncommon Variant of Guillain-Barre
Syndrome. Journal of clinical and diagnostic research: JCDR. 2016 Jul;10(7):OD01. 